Gynäk. Rdsch. 1981;21(Suppl. 2):85-86

\title{
Mikrochirurgische Behandlung des Tubenverschlusses
}

\begin{tabular}{|l|l|}
\hline W. & Albrich \\
\hline A. & Götz \\
\hline
\end{tabular}

Frauenklinik der Universität Miinchen im Klinikum Grosshadern (Direktor: Prof. Dr. $K$. Richter), München, BRD

Dr. W. Albrich, Frauenklinik im Klinikum Grosshadern, Marchioninistrasse 15, D-8000 München 70 (BRD)

Die Einführung des Operationsmikroskops in die Gynäkologie ist Walz [1959] zu verdanken.

Die Verbreitung durch angloamerikanische Gynäkologen erfolgte mehr als 15 Jahre später

[Gomel, 1977; Winston, 1977]. Die inzwischen mitgeteilten Ergebnisse in der Literatur und eigene Erfahrungen bei mehr als 100 mikrochirurgischen Eingriffen geben Anlass zu nüchterner Betrachtung des Verfahrens.

Die Fortschritte der Mikrochirurgie beruhen auf wählbarer optischer Vergrösserung, atraumatischen Instrumenten, feinstem Nahtmaterial, Feuchthalten des Operationsfeldes und sorgfältigster elektrochirurgischer Blutstillung. Die präoperative Diagnostik umfasst die üblichen Untersuchungen: Basaltemperatur, Spermiogramm, Postkoitaltest, Laparo-skopie und gegebenenfalls Hysterosalpingographie. Die Kontraindikationen entsprechen denen konventioneller Sterilitätsoperationen: Genitaltuberkulose, akute Entzündung, schwere Allgemeinerkrankungen und Erbkrankheiten. Schlechte Ergebnisse sind zu erwar-ten bei Tubenlängen unter $4 \mathrm{~cm}$ und gleichzeitigem proximalem und distalem Tubenver-schluss. Von den Kontraindikationen sind nicht operationswürdige Befunde zu unterschei-den.

Technik

Zur mikrochirurgischen Operation wird der Douglas-Raum mit feuchten Kompres-sen ausgestopft. Die dadurch elevierten Adnexe werden durch eine speziell geformte Sili-konplatte stabilisiert. Unter dem Operationsmikroskop erfolgt die Adhäsiolyse elektrochir-urgisch mit feinsten Nadeln. Anastomosen werden zweischichtig extramukös genäht. Bei Salpingostomien kann durch die optische Vergrösserung die Schleimhauttextur berücksich-tigt werden. Die Spülung mit physiologischen Lösungen halt das Operationsfeld feucht und erleichtert das Erkennen winziger blutender Gefässe, die bipolar elektrokoaguliert werden.

Krankengut

In den letzten 3 Jahren wurden an unserer Klinik 109 mikrochirurgische Eingriffe durchgeführt. 23mal wurden Adhäsiolysen oder Salpingoplastiken durchgeführt, 39 mal Salpingotomien und 12 mal Anastomosen zur Refertilisierung oder bei entzündlichen Ver-schlüssen. Einer 4. Gruppe ordneten wir 35 Operationen zu für die eine statistische Aus-wertung nicht möglich ist: seitenverschiedene Eingriffe, kombinierte Operationen bei ampullärem und isthmischem Verschluss und konservative Operationen bei Tubargravidi-tät.

Mitteilungen zum 2. Hauptthema

86

Ergebnísse 
Da nur knapp 70 Operationen länger als 1 Jahr zurückliegen, haben die Ergebnisse nur vorläufigen Aussagewert. Die 11 intrauterinen Schwangerschaften verteilten sich auf alle 4 Operationsgruppen. 5 weitere Schwangerschaften waren extrauterin und traten 1-19 Monate nach der Operation ein.

Diskussion

Als erfolgslimitierend erweist sich der meist schwere postentzündliche Schaden an Tubenmukosa und -muskularis. Erst bei Refertilisierungen - der Anastomose unterbunde-ner gesunder Tuben - wird der gewebsschonende Effekt der Mikrochirurgie auch an den Erfolgen statistisch erkennbar: Mit konventionellen Operationen werden Schwanger-schaftsraten von 1350\% erreicht, mit mikrochirurgischen Anastomosen 60-80\%. Diese hohe Erfolgsrate rechtfertigt nach unserer Meinung den grossen Aufwand der Mikrochirurgie.

Literatur

Gomel, V.: Tubal reanastomosis by microsurgery. Fert. Steril. 28: 59-65 (1977).

Walz, W.: Sterilitätsoperationen an der Tube mit Hilfe eines Operationsmikroskopes. Z.

Geburtsh. Gynäk. 153: 49-55 (1959).

Winston, R.M.L.: Microsurgical tubocornual anastomosis for reversal of sterilisation. Lancet i: 284-285 (1977).

Gynäk. Rdsch. 21 (Suppl. 2): 86-89 (1981)

Ergebnisse der mikrochirurgischen Tubenrekonstruktion

P. Scheidel, H. Hepp

Universitäts-Frauenklinik des Saarlandes (Direktor: Prof. Dr. H. Hepp), Homburg/Saar, BRD

Die Kenntnisse über Physiologie und Pathologie des Eileiters sind nach wie vor zu gering, um die komplexen Vorgänge des Eiauffangmechanismus und des Eitransports bzw. Störungen nach entzündlichen Veränderungen hinlänglich erklären zu können.

Noch 1978 überschrieb Pauerstein [1] eine Übersichtsarbeit mit dem Titel «From Fallopius to Fantasy», um klar darzustellen, wieviele unserer Vorstellungen über dieGynäk. Rdsch. 21

(Suppl. 2): 85-86 (1981) 